\title{
On the diurnal cycle and susceptibility to aerosol concentration in a stratocumulus-topped mixed layer
}

\author{
By YUNYAN ZHANG ${ }^{1,2 *}$, BJORN STEVENS ${ }^{1}$ and MICHAEL GHIL ${ }^{1,2,3}$ \\ ${ }^{1}$ Department of Atmospheric and Oceanic Sciences, University of California at Los Angeles, USA \\ ${ }^{2}$ Institute of Geophysics and Planetary Physics, University of California at Los Angeles, USA \\ ${ }^{3}$ Département Terre-Atmosphère-Océan and Laboratoire de Météorologie Dynamique du CNRS/IPSL, \\ Ecole Normale Supérieure, France
}

(Received 5 July 2004; revised 22 November 2004)

\section{SUMMARY}

Mixed-layer theory is used to study the diurnal cycle of the stratocumulus-topped boundary layer and its susceptibility to perturbations in atmospheric aerosol concentration. Our results show that the diurnal evolution of cloud thickness is sensitive to the entrainment efficiency. For high entrainment efficiencies, the cloud base tends to descend at a faster rate than the cloud top; this difference in descent rates leads to cloud thickening during the daytime, which is inconsistent with observations. For low entrainment efficiencies, variations in cloud-top height dominate in the cloud-thickness evolution, while cloud-base height remains almost constant; this behaviour is in better agreement with available data. We explain these effects through a consideration of the equilibrium state of cloud boundaries and their adjustment time-scales. Liquid-water path and cloud albedo are both sensitive to the entrainment efficiency; still, the susceptibility of cloud albedo to droplet number density dominates the entrainment effects. This result has significant implications for climate-sensitivity studies: it suggests that estimates of aerosol indirect effects from stratocumulus clouds will not be particularly sensitive to the way entrainment is represented in large-scale models.

KEYwords: Aerosol indirect effect Cloud albedo Entrainment

\section{INTRODUCTION}

Stratocumulus is a long-lasting low cloud type that covers a wide area and is usually observed in maritime environments, eastward and equatorward of high pressure zones in the subtropics. This cloud type has a high albedo relative to the underlying ocean surface and hence plays an important role in modulating local meteorology and global climate.

The mixed-layer model (MLM) introduced by Lilly (1968) provides the theoretical framework upon which most of our understanding of the marine stratocumulus-topped boundary layer (STBL) is based. It has been implemented directly in a number of general circulation models (e.g. at the University of California at Los Angeles and Colorado State University). An increasing number of climate models (e.g. UKMO, GFDL, NCAR-CAM) are either adopting, or experimenting with, parametrizations that attempt to mimic the behaviour of the STBL (Grenier and Bretherton 2001; Lock 2001) in certain regimes. Even so, this model has been called into question as unable to adequately represent several major aspects of the STBL diurnal cycle.

Schubert (1976) (hereafter S76) showed that the diurnal cycle in the MLM tends to produce clouds that are thicker during the day and thinner at night than observed. Specifically, in Schubert's results the cloud base is shown to descend during the day at a rate somewhat greater than that of the cloud top. Observations have shown, however, that the diurnal evolution of marine stratocumulus is characterized by an ascending cloud base, not a descending one. Although previous observations (Vernon 1936; Hignett 1991) may be questioned, given the varied influences of land (in the case of Vernon) or island effects (in the case of Hignett), recent shipboard observations taken in October

* Corresponding author: Department of Atmospheric and Oceanic Sciences, University of California at Los Angeles, 405 Hilgard Ave., Box 951565, Los Angeles, CA 90095-1565. e-mail: yunyan@ atmos.ucla.edu

(C) Royal Meteorological Society, 2005. 
2001 in the south-eastern Pacific during the East Pacific Investigation of Climate (EPIC) also show little evidence of the cloud base descending through the day (Bretherton et al. 2004). Well away from land, the cloud base thus appears to be relatively constant in height throughout the diurnal cycle, with most of the variation being evident at cloud top.

This discrepancy between the MLM results and the observations has fuelled the speculation that decoupling between the cloud layer and the surface plays an essential role in the dynamics of the STBL. According to this thought, solar heating during the daytime leads to the formation of a stable layer near the cloud base, which isolates the cloud layer from the moisture source at the surface and causes it to thin under the influence of entrainment (Turton and Nicholls 1987). This idea, too, seems inconsistent with the EPIC data, wherein the observed boundary layers appeared to remain relatively well mixed throughout the diurnal cycle.

These difficulties in reconciling MLM results with observations might be related to the fact that the entrainment parametrization used by S76 tends to overestimate the entrainment rates at the top of stratocumulus clouds. Indeed, Schubert's parametrization of entrainment was formulated in a way which precludes the study of the relatively weak entrainment efficiencies that appear to be more representative of actual clouds (Lewellen and Lewellen 1998, hereafter LL98; vanZanten et al. 1999; Stevens 2002). The study by Turton and Nicholls (1987) shows that the extent of decoupling is sensitive to the representation of the entrainment. Recent study by Duynkerke et al. (2004) also indicates that different entrainment rate may result in different evolution of stratocumulus liquid-water path. All these suggest that the puzzling behaviour of the MLM diurnal cycle might be an artifact of the entrainment parametrization employed. We shall see that the diurnal cycle does show marked sensitivity to entrainment efficiency, with more realistic entrainment rates leading to a more realistic diurnal cycle.

This finding in turn motivates the broader question of how sensitive stratocumulus variability is to the details of the entrainment parametrization. In addressing this question we focus not only on the diurnal cycle, but also on the susceptibility of stratocumulus albedo to perturbations in the ambient aerosol concentration.

Aerosols impact climate through their direct effect on the radiation balance. They also have an indirect effect on climate by affecting the formation, maintenance and dissipation of clouds. Twomey (1977) suggested that increased air pollution enhances cloud albedo; this is often called the first indirect effect. Previous studies have shown that, in addition to the Twomey effect, stratocumulus optical properties are sensitive to the character of the ambient aerosol concentration through the modification of the cloud macrostructure by droplet number density. This latter effect is referred to as the second indirect effect. Studies emphasizing this second effect (Albrecht 1989; Boers and Mitchell 1994; Pincus and Baker 1994) have largely been based on a bulk parametrization of the STBL or an MLM frameworks. These estimates for microphysics-related cloud properties might also be influenced by what assumptions one makes about the entrainment rate.

To address these issues, we revisit the diurnal cycle of the STBL. The MLM we use is Stevens's (2002) version of Lilly's (1968) model; its present implementation incorporates time-varying forcing and is briefly described in section 2 . In section 3 , we introduce the entrainment parametrization based on the process partitioning used to explain the large-eddy simulation results of several groups (e.g. LL98; vanZanten et al. 1999) and compare this parametrization with the one of S76. In section 4, the model results of STBL diurnal evolution, given a diurnally varying radiative driving, are presented and explained; these results confirm that entrainment parametrization may 
artificially affect STBL dynamics. Based on these results, we investigate in section 5 the radiative properties of stratocumulus clouds, such as the susceptibility of the cloud albedo to droplet number density changes. Conclusions are drawn in section 6 .

\section{MIXED-LAYER MODEL}

The STBL is assumed to be well-mixed throughout its depth and possess horizontally homogeneous thermodynamic fields. Mixing is mainly driven by radiative cooling at the cloud top. For a given surface pressure, the state of this bulk layer is uniquely determined by its depth $h$ (also the height of the mixed layer and the cloud top), liquid-water static energy $s_{\ell}$ and total-water specific humidity $q_{\mathrm{t}}$. The latter two are approximately invariant following isentropes in the moist system and are defined as follows:

$$
\begin{aligned}
& s_{\ell}=c_{p} T+g z-L_{\mathrm{v}} q_{\ell}, \\
& q_{\mathrm{t}}=q_{\mathrm{v}}+q_{\ell},
\end{aligned}
$$

where $c_{p}$ is the isobaric specific heat capacity, $T$ the air temperature, $g$ the gravity, $z$ the height above the surface, $L_{\mathrm{v}}$ the enthalpy of vaporization, $q_{\ell}$ the liquid-water specific humidity and $q_{\mathrm{v}}$ the water-vapour specific humidity.

The prognostic equations for $h, s_{\ell}$ and $q_{\mathrm{t}}$ are:

$$
\begin{aligned}
\frac{\mathrm{d} h}{\mathrm{~d} t} & =E-D h, \\
h \frac{\mathrm{d}}{\mathrm{d} t}\left\langle s_{\ell}\right\rangle & =V\left(s_{\ell, 0}-\left\langle s_{\ell}\right\rangle\right)+E\left(s_{\ell,+}-\left\langle s_{\ell}\right\rangle\right)-\frac{\Delta F_{s}}{\rho}, \\
h \frac{\mathrm{d}}{\mathrm{d} t}\left\langle q_{\mathrm{t}}\right\rangle & =V\left(q_{\mathrm{t}, 0}-\left\langle q_{\mathrm{t}}\right\rangle\right)+E\left(q_{\mathrm{t},+}-\left\langle q_{\mathrm{t}}\right\rangle\right)-\frac{\Delta F_{q}}{\rho} .
\end{aligned}
$$

In the above

$$
\langle X\rangle=\frac{1}{h} \int_{0}^{h} x \mathrm{~d} z
$$

stands for the vertically averaged, or bulk value, and $\mathcal{X} \in\left(s_{\ell}, q_{\mathrm{t}}\right)$. Subscripts 0 and + denote surface values and the states just above cloud top, respectively, $\rho$ is the air mass density, while $\Delta F_{s}$ and $\Delta F_{q}$ are the diabatic forcings. Specifically, $\Delta F_{s}$ consists of the radiative driving, $\Delta F_{R}$, and the drizzle effect on liquid-water content in $s_{\ell}, \Delta F_{q}$ is the drizzle effect on $q_{\mathrm{t}}$, and $D$ represents the large-scale divergence. Surface fluxes are calculated by a bulk aerodynamic formula, where $V=C_{D}\|U\|$, with $\|U\|$ the surface wind speed, and $C_{D}$ the surface exchange coefficient, which is assumed constant. Equation (3) essentially defines $E$, the entrainment rate, as the diabatic growth rate of the mixed-layer depth. To close the system for a given set of equilibrium conditions requires the specification of $E$; this is done in section 3 later.

A diagnostic measure of the state of the cloud is the liquid-water path $L$, defined as:

$$
L=\int_{b}^{h} \rho q_{\ell} \mathrm{d} z,
$$

where $b$, the cloud-base height, is an implicit function of $s_{\ell}, q_{\mathrm{t}}$ and the surface pressure. In the cloud layer, $q_{\ell}$ is assumed to increase linearly with height, according to observations. Therefore $L$ is proportional to $(h-b)^{2}$. Furthermore, the liquid-water path is also important in studying cloud radiative properties (Stephens 1978a,b; Liou 2002). We thus focus hereafter on $L$ instead of simply cloud thickness. 


\section{ENTRAINMENT PARAMETRIZATION}

An entrainment parametrization is needed to close the system (3)-(5). Physically, entrainment is related to the production of turbulent kinetic energy (TKE) and the stability of the interfacial layer. The TKE for stratocumulus depends on the strength of forcing, while the interfacial layer is the very thin undulating layer between the heights just above and just below the cloud top.

In this study, two entrainment parametrizations are investigated: one following S76, and the other following LL98. S76 obtains a value for $E$ by constructing the buoyancy flux profile as follows:

$$
\mathcal{B}_{\min }=-\frac{2 k}{1-k}\langle\mathscr{B}\rangle,
$$

where usually $k=0.2, \mathscr{B}_{\min }$ is the vertical minimum of the buoyancy flux, and $\langle\mathscr{B}\rangle$ is the vertically averaged value of $\mathscr{B}$, as defined in (6) of the previous section.

S76 connects the minimum buoyancy flux to the averaged vertical integral of this flux, which is proportional to the TKE generation. Because $\mathcal{B}$ is a function of $E$, (8) defines $E$ implicitly, allowing one to solve for it given $k$.

For $k>0$, the minimum buoyancy flux is always non-positive. For quasi-steady states, given constant radiative driving, a negative buoyancy is most likely at cloud top or just below cloud base. Concerning the first location, we assume the radiative driving occurs in a very thin layer at the cloud top. As this radiative length-scale becomes infinitesimally small, the non-positive buoyancy flux approaches zero. Whether $\mathscr{B}_{\min }$ is located at cloud top or not depends on how the radiative driving is specified; hence (8) determines $E$ most robustly when $\mathscr{B}_{\min }$ is associated with the value of $\mathscr{B}$ just below cloud base. Requiring $\mathscr{B}$ to be negative in the subcloud layer is, however, a strong assumption since it is reasonable to assume that entrainment is sufficiently weak for $\mathscr{B}$ to be positive everywhere in the STBL. Previous studies (Bretherton and Wyant 1997; Stevens 2000) have indeed shown that negative $\mathscr{B}$ in the subcloud layer implies that other sources of TKE, such as strong surface buoyancy fluxes, are significant compared to radiative cooling. In our study, this situation is more likely to occur around noon, when infrared (IR) cooling is partially abated by solar heating. This quasi-equilibrium behaviour of the STBL implies that, given variable radiative driving, the corresponding variation in $E$ will also be exaggerated by S76.

In the LL98 parametrization, the single control parameter is the entrainment efficiency $\eta$,

$$
\eta=\int_{0}^{h}\left(\mathscr{B}_{\mathrm{NE}}-\mathcal{B}\right) \mathrm{d} z / \int_{0}^{h} \mathscr{B}_{\mathrm{NE}} \mathrm{d} z,
$$

where $\mathscr{B}$ is the buoyancy flux, and $\mathscr{B}_{\mathrm{NE}}$ is its value if there is no entrainment. The limits of integration represent the surface $(z=0)$ and the STBL depth $(z=h)$, respectively. The advantage of this parametrization is that varying $\eta$ between 0 and 1 samples the full range of entrainment efficiencies. This is in contrast to the S76 closure, wherein the variation of the entrainment parameter $k$ in (8) between 0 and 1 samples only a range of entrainment efficiencies that corresponds roughly to $0.6<\eta<1.0$.

In LL98, the minimum buoyancy flux $\mathcal{B}_{\min }$ is not necessarily non-positive. Furthermore LL98 does not make any assumption on the net buoyancy flux profile. With (9), LL98 directly relates the consumption of TKE by entrainment to the production of TKE by other processes. Using the LL98 parametrization with $\eta=0.25$, Stevens et al. (2003a) have produced reasonable model results compared with the observations 


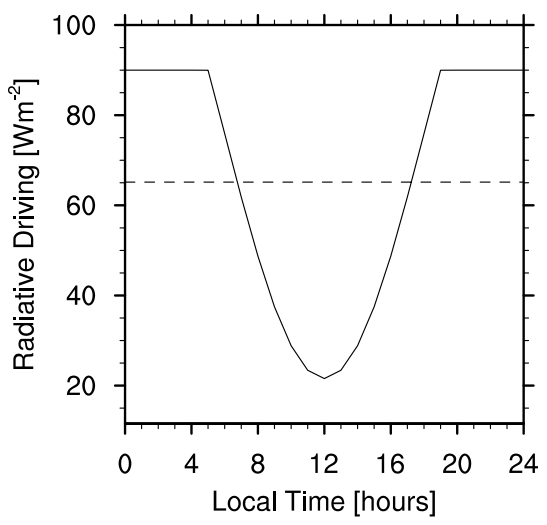

Figure 1. Diurnal variation of $\Delta F_{R}$, the radiative driving at cloud top. Dashed line illustrates the daily average.

from the first research flight (RF01) of the second field study on the DYnamics and Chemistry Of Marine Stratocumulus (DYCOMS-II) (Stevens et al. 2003b).

The model results produced by the $\mathrm{S} 76$ entrainment rule (8) with $k=0.2$ are quite similar to the LL98 results with $\eta=0.77$. This comparison justifies our statement that the S76 rule samples only a limited range of entrainment efficiencies, namely high values. To simplify the analysis we take advantage of this result and use LL98 with $\eta=0.77$ to substitute for S76 with $k=0.2$ in the remainder of this study: our arguments can thus be expressed entirely in terms of entrainment efficiencies. Although not shown, we have checked that any conclusion drawn based on LL98 with $\eta=0.77$ does indeed hold for the S76 rule with $k=0.2$.

\section{STBL DIURNAL VARIATION}

In modelling the diurnal cycle, we follow S76 and assume the STBL is nonprecipitating and evolves through a succession of quasi-steady states, as described by Stevens (2002). Therefore, in (4) and (5), $\Delta F_{q}=0$ and $\Delta F_{s}$ equals simply $\Delta F_{R}$, the radiative driving. IR (also referred to as long-wave) cooling and solar (also referred to as short-wave) heating both occur in fact through certain finite-depth ranges in the cloud layer, but we assume here that the net radiative driving is located only at cloud top; using this assumption is consistent with the boundary projection method of Stevens (2002). Diurnal variations in large-scale subsidence, as well as in ocean surface temperatures, may also affect the diurnal evolution of cloudiness, but we focus here only on the effect of the diurnal variation in the radiative driving.

The cyclic pattern of the radiative driving is chosen, for reasons of consistency, to follow S76, with sunrise at 0500 LST (LST denotes model hours since local solar midnight) and sunset at 1900 LST (see Fig. 1). Due to the combined effect of IR cooling and solar heating, $\Delta F_{R}$ decreases from dawn to noon, reaching a minimum of $20 \mathrm{~W} \mathrm{~m}^{-2}$. It then increases from noon till dusk. At night only outgoing IR cooling is present, so $\Delta F_{R}$ stays constant at $90 \mathrm{~W} \mathrm{~m}^{-2}$. Prescribed large-scale conditions are taken from DYCOMS-II (Stevens et al. 2003b) and listed in Table 1.

To illustrate the sensitivity of the simulated STBL diurnal cycle to the entrainment efficiency, we investigate two cases: one with $\eta=0.20$ and the other with $\eta=0.77$. Initial conditions are listed in Table 2 . Each experiment is started from its equilibrium corresponding to $\Delta F_{R}=65 \mathrm{~W} \mathrm{~m}^{-2}$, the daily average of $\Delta F_{R}$. 
TABLE 1. BOUNDARY CONDITIONS

\begin{tabular}{lc}
\hline Large-scale divergence $(D)$ & $6 \times 10^{-6} \mathrm{~s}^{-1}$ \\
Surface wind speed $(\|U\|)$ & $7 \mathrm{~m} \mathrm{~s}^{-1}$ \\
Sea surface temperature & $290 \mathrm{~K}$ \\
Specific humidity above cloud $\left(q_{\mathrm{t},+}\right)$ & $3.5 \mathrm{~g} \mathrm{~kg}^{-1}$ \\
\hline
\end{tabular}

TABLE 2. INITIAL CONDITIONS

\begin{tabular}{ccc}
\hline Variable & $\eta=0.77$ & $\eta=0.20$ \\
\hline$h$ & $1002.5 \mathrm{~m}$ & $717.5 \mathrm{~m}$ \\
$\theta_{\ell} \approx s_{\ell} / c_{p}$ & $291 \mathrm{~K}$ & $288 \mathrm{~K}$ \\
$q_{\mathrm{t}}$ & $8.2 \mathrm{~g} \mathrm{~kg}^{-1}$ & $8.9 \mathrm{~g} \mathrm{~kg}^{-1}$ \\
\hline
\end{tabular}

See text for explanation of symbols.
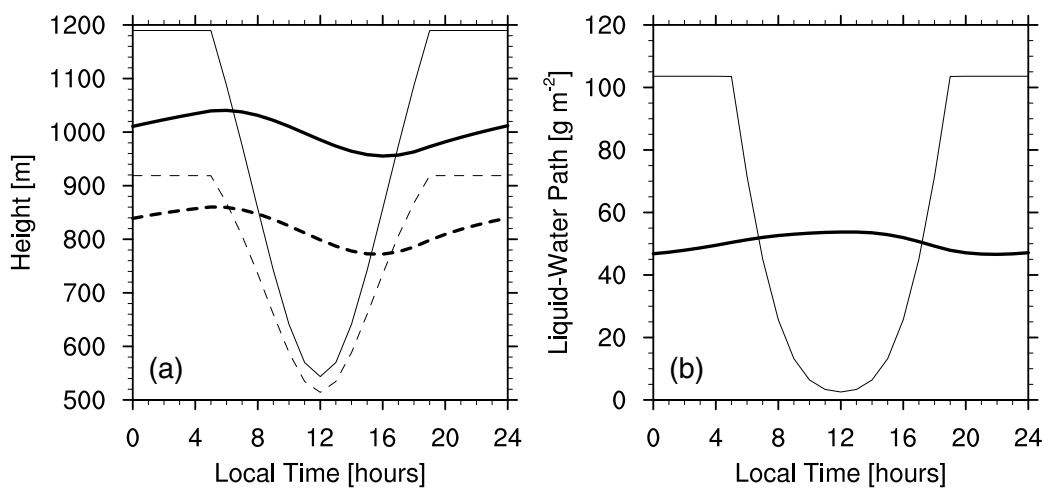

Figure 2. MLM results for the STBL diurnal variation when using the LL98 entrainment rule with $\eta=0.77$. (a) Transient mixed-layer depth $h$ (heavy solid line) and its equilibrium solution $h_{\mathrm{e}}$ (light solid line); transient cloud base $b$ (heavy dashed line) and its equilibrium solution $b_{\mathrm{e}}$ (light dashed line). (b) Transient liquid-water path $L$ (heavy solid line) and its equilibrium solution $L_{\mathrm{e}}$ (light solid line). These results represent a good approximation for the model's behaviour when using S76 closure with $k=0.2$ (not shown). See text for further explanation.

Figure 2 illustrates the diurnal cycle for the model using the LL98 closure with $\eta=0.77$, while the case with $\eta=0.20$ is shown in Fig. 3. The transient solutions (heavy curves in both figures) show the results for the full time-dependent calculation using (3)-(5) with diurnally varying radiative driving, while the equilibrium solutions (light curves) indicate equilibrium calculations in which MLM variables are allowed to fully adjust to the instantaneous value of the radiative driving at each time of day. The shallower boundary layer and thicker cloud in Fig. 3 reflect the effectively weaker entrainment for $\eta=0.20$ (cf. Stevens 2000).

In both Figs. 2 and 3 the transient cloud-top height $h$ achieves its maximum around 0600 LST and its minimum around 1700 LST. With $\eta=0.77$, however, $h$ is larger and also has a larger diurnal variation than for the case with $\eta=0.20$. In Fig. 2 the transient cloud-base height $b$ exhibits a marked diurnal variation with an amplitude of about $90 \mathrm{~m}$. In contrast, in Fig. 3 the transient $b$ remains relatively constant, which is more consistent with the EPIC data (Bretherton et al. 2004). With $\eta=0.77$, the transient $L$ increases 

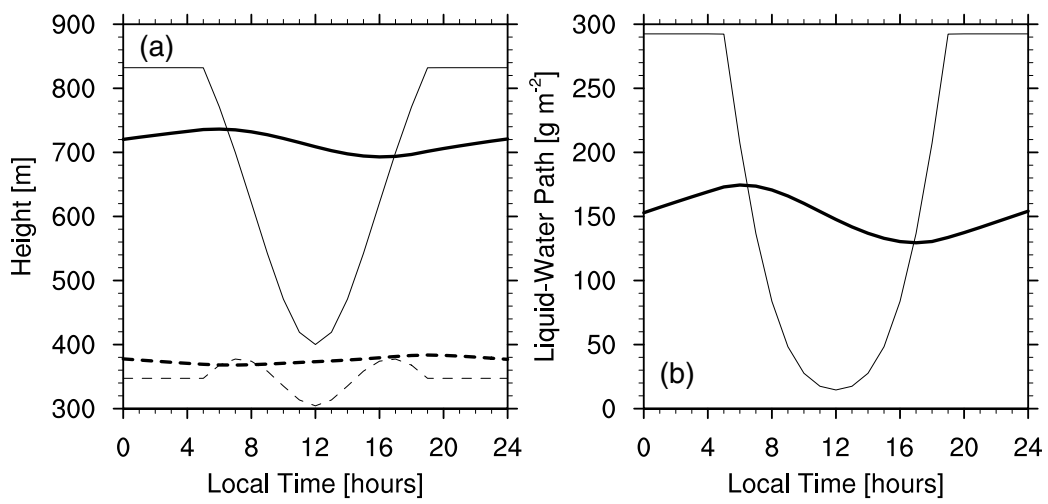

Figure 3. As in Fig. 2, but with $\eta=0.20$.

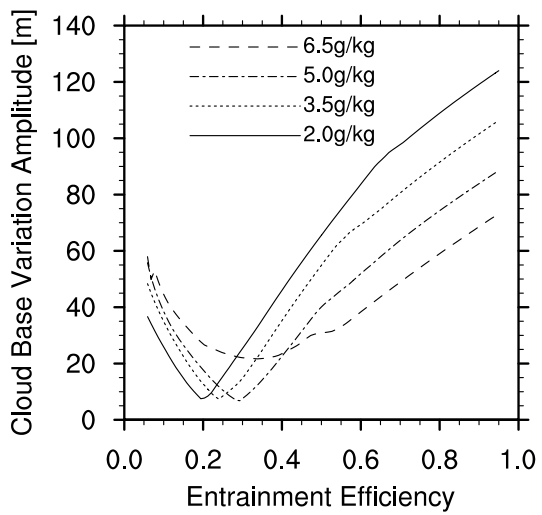

Figure 4. Diurnal variation in amplitude (maximum minus minimum) of cloud base height $b$ as a function of entrainment efficiency $\eta$, for several values of free-atmosphere specific humidity $q_{\mathrm{t},+}$; these values are shown in the legends.

during the day and is in phase opposition with its expected equilibrium behaviour. For $\eta=0.20, L$ lags its equilibrium response by 6 hours approximately.

Such differences are found not only between these two specific cases, but across the full range of entrainment efficiencies. Figure 4 shows the variation in amplitude of cloud-base height $b$ as a function of entrainment efficiency $\eta$ for several values of the free-atmosphere specific humidity $q_{\mathrm{t},+}$. The amplitude varies more rapidly with $\eta$ than with $q_{\mathrm{t},+}$. Along constant $q_{\mathrm{t},+}$, the amplitude first decreases and then increases with $\eta$. The position of the minimum in amplitude shifts to slightly larger values of $\eta$ as $q_{\mathrm{t},+}$ increases and the minimum becomes shallower.

Clearly entrainment efficiency has a pronounced effect on the STBL's diurnal evolution. What causes these differences? The equilibrium solutions for $h, b$, and $L$, also plotted in Figs. 2 and 3, help us address this question. The equilibrium solutions for $L$ are very similar in the two cases of $\eta=0.77$ and $\eta=0.20$ : both show a pronounced minimum when radiative driving is at its minimum. This similarity implies that, if the MLM were always in instantaneous equilibrium with its forcing, the diurnal cycle of $L$ would be relatively insensitive to the entrainment closure. Consequently, the 
differences in transient behaviour, in which the phase of the STBL diurnal cycle shifts with entrainment efficiency, must result from differences in the adjustment processes.

To explore this idea, it is useful to introduce a simplified system of equations, in which the transient variations follow the changes in the equilibrium state of the STBL:

$$
\begin{aligned}
\frac{\mathrm{d} h}{\mathrm{~d} t} & =-\frac{h-h_{\mathrm{e}}(t)}{\tau_{h}}, \\
\frac{\mathrm{d}\left\langle s_{\ell}\right\rangle}{\mathrm{d} t} & =-\frac{\left\langle s_{\ell}\right\rangle-\left\langle s_{\ell}\right\rangle_{\mathrm{e}}(t)}{\tau_{s}}, \\
\frac{\mathrm{d}\left\langle q_{\mathrm{t}}\right\rangle}{\mathrm{d} t} & =-\frac{\left\langle q_{\mathrm{t}}\right\rangle-\left\langle q_{\mathrm{t}}\right\rangle_{\mathrm{e}}(t)}{\tau_{q}},
\end{aligned}
$$

where subscript e stands for the equilibrium solution. Note that $h_{\mathrm{e}},\left\langle s_{\ell}\right\rangle_{\mathrm{e}}$ and $\left\langle q_{\mathrm{t}}\right\rangle_{\mathrm{e}}$ are functions of time and evolve with $\Delta F_{R}$, while $\tau_{h}, \tau_{s}$ and $\tau_{q}$ are the adjustment time-scales for $h,\left\langle s_{\ell}\right\rangle$ and $\left\langle q_{\mathrm{t}}\right\rangle$, respectively. As shown by Schubert et al. (1979), the magnitude of $\tau_{h}$ is approximately one week, which is determined by large-scale subsidence, while $\tau_{s}$ and $\tau_{q}$ both equal about one day, which is determined by the STBL thermodynamics; $\tau_{h}$ is thus generally much larger than $\tau_{s}$ and $\tau_{q}$.

In the mixed-layer framework, $L$ essentially represents $(h-b)$. What controls the transient $h$ ? As shown in Figs. 2 and 3, at night, the STBL is in a constant equilibrium state, which corresponds to the fixed IR cooling of $90 \mathrm{~W} \mathrm{~m}^{-2}$ (see Fig. 1). After dawn, solar heating abates IR cooling and $h_{\mathrm{e}}$ changes rapidly with time. Note that, in Figs. 2 and 3 , the sign of $\mathrm{d} h / \mathrm{d} t$ is indeed given by the sign of $\left(h_{\mathrm{e}}-h\right)$, as would be expected if $h(t)$ were actually governed by (10). This adjustment causes $h$ to lag $h_{\mathrm{e}}$ by several hours.

What controls the transient $b$ ? In our MLM, the cloud-base height is diagnosed from the total specific humidity and the air temperature, which can be derived from the liquid-water static energy. Therefore $b$ adjusts to its equilibrium value $b_{\mathrm{e}}$, with a timescale that is determined by that of the thermodynamic quantities, $\tau_{s}$ and $\tau_{q}$. In Fig. 2, the diurnal cycle of $b_{\mathrm{e}}$ is very similar to that of $h_{\mathrm{e}}$. Although $\left(h-h_{\mathrm{e}}\right)>\left(b-b_{\mathrm{e}}\right)$, the adjustment time-scale $\tau_{h}$ is much longer than $\tau_{s}$ and $\tau_{q}$; consequently the cloud base descends more rapidly than the cloud top, causing the layer to thicken as it adjusts to the changing forcing. This thickening is seen even better for the liquid-water path in Fig. 2(b); in Fig. 2(a), the vertical scale is chosen to show the equilibrium solutions and so it does not illustrate this more rapid descending of cloud base very well. In contrast $b_{\mathrm{e}}$ shows a weak diurnal variation in Fig. 3. In this case, $\left|b-b_{\mathrm{e}}\right|$ is so small that $|\mathrm{d} b / \mathrm{d} t|<|\mathrm{d} h / \mathrm{d} t|$. Thus the transient $b$ is very nearly constant, and hence the variation in $h$ dominates the diurnal evolution of $L$.

Two distinct processes are evident in the STBL behaviour discussed above. First, $h$ and $b$ adjust to their equilibria on different time-scales. Second, $b_{\mathrm{e}}$ varies significantly with the entrainment parametrization being used. The equilibrium solutions and the adjustment time-scales together determine the evolution of $L$.

To understand more clearly the STBL behaviour in Figs. 2 and 3, it is useful to ask how $\eta$ influences $b_{\mathrm{e}}$. To make the problem analytically tractable, we define a radiative entrainment efficiency $\alpha$ :

$$
\alpha=\frac{E \Delta s_{\ell}}{\Delta F_{R}},
$$

where $\Delta s_{\ell}=s_{l,+}-\left\langle s_{\ell}\right\rangle$. The value of $\alpha$ depends, of course, on the entrainment rule and it is not constant in general. By investigating solutions at constant $\alpha$, we can, however, develop insight into how $\eta$ affects the equilibrium state. 
Given (13), $h_{\mathrm{e}},\left\langle s_{\ell}\right\rangle_{\mathrm{e}}$ and $\left\langle q_{\mathrm{t}}\right\rangle_{\mathrm{e}}$ can be written as:

$$
\begin{aligned}
h_{\mathrm{e}} & =h_{0}\left(\frac{\alpha}{1+\sigma-\alpha}\right), \\
\left\langle s_{\ell}\right\rangle_{\mathrm{e}} & =s_{\ell, 0}-\left(s_{\ell,+}-s_{\ell, 0}\right)\left(\frac{1-\alpha}{\sigma}\right), \\
\left\langle q_{\mathrm{t}}\right\rangle_{\mathrm{e}} & =q_{\mathrm{t}, 0}+\left(q_{\mathrm{t},+}-q_{\mathrm{t}, 0}\right)\left(\frac{\alpha}{1+\sigma}\right),
\end{aligned}
$$

with $\sigma=V\left(s_{\ell,+}-s_{\ell, 0}\right) / \Delta F_{R}$. Here $h_{0}=V / D ;\left(s_{\ell,+}-s_{\ell, 0}\right)$ represents the lower troposphere stability, which is relatively constant and positive, while the value of $\left(q_{\mathrm{t},+}-q_{\mathrm{t}, 0}\right)$ is usually negative.

If $\alpha$ remains constant, $\sigma$ captures the diurnal cycle of $\Delta F_{R}$ in the equilibrium solutions. At night, only IR cooling is present so $\sigma$ is fixed, and the equilibrium solutions remain constant. In the morning, $\Delta F_{R}$ begins to decrease and thus $\sigma$ increases, leading to an increase in $\left\langle s_{\ell}\right\rangle_{\mathrm{e}}$ and $\left\langle q_{\mathrm{t}}\right\rangle_{\mathrm{e}}$, and a decrease in $h_{\mathrm{e}}$. Cloud base rises as the STBL warms and dries. Increases in both $\left\langle s_{\ell}\right\rangle_{\mathrm{e}}$ and $\left\langle q_{\mathrm{t}}\right\rangle_{\mathrm{e}}$ have counteracting influences on cloud-base height. In the afternoon, solar heating begins to decrease and the evolution is reversed.

For different entrainment efficiencies, however, the diurnal evolution and effect of $\alpha$ differs. In general, $\alpha$ is larger for higher entrainment efficiencies and smaller for lower ones. A diurnal evolution in $\alpha$ is to be expected, given the differing degree to which $\Delta F_{R}$ contributes to the TKE production throughout the course of the diurnal cycle, but we neglect this evolution for the moment.

From (15)-(16), the amplitude of the diurnal cycle in $b_{\mathrm{e}}$ depends on $\alpha$. We consider two limits: $\alpha=1$ for high entrainment efficiency and $\alpha=0$ for low entrainment efficiency. As $\alpha$ becomes large, the diurnal cycle of $\left\langle s_{\ell}\right\rangle_{\mathrm{e}}$ will be diminished, while that of $\left\langle q_{\mathrm{t}}\right\rangle_{\mathrm{e}}$ is enhanced. When $\alpha=1,\left\langle s_{\ell}\right\rangle_{\mathrm{e}}$ will remain constant throughout the day, while the diurnal cycle in $\left\langle q_{\mathrm{t}}\right\rangle_{\mathrm{e}}$ dominates the diurnal variations; this will result in a lowering of $b_{\mathrm{e}}$. As $\alpha$ approaches 0 , the diurnal cycle of $\left\langle q_{\mathrm{t}}\right\rangle_{\mathrm{e}}$ disappears, while the diurnal effect of $\left\langle s_{\ell}\right\rangle_{\mathrm{e}}$ is amplified; this will result in a rising of $b_{e}$.

This discussion suggests that $\alpha$ mediates between the competing effects of $\left\langle s_{\ell}\right\rangle_{\mathrm{e}}$ and $\left\langle q_{\mathrm{t}}\right\rangle_{\mathrm{e}}$ on $b_{\mathrm{e}}$. Overestimating entrainment produces a significant increase in the diurnal variation of the cloud base, especially in its lowering. This effect is also evident in Fig. 4.

The LL98 rule generates a pronounced diurnal variation in $\alpha$, which we have neglected so far. To see what role this variation plays, we examine $\alpha$ in two cases: $\eta=0.77$ and $\eta=0.20$ (see Fig. 5). The mean value of $\alpha$ is smaller for $\eta=0.20$ than for $\eta=0.77$; furthermore a more pronounced diurnal evolution in $\alpha$ occurs-especially during the daytime, when the sun shines-for $\eta=0.20$ than for $\eta=0.77$.

What then makes the weak-entrainment results more reasonable- the smaller mean value of $\alpha$ or the larger diurnal variation of $\alpha$ ? To answer this question we simulate the diurnal cycle using the highly simplified entrainment rule (13) and substitute the mean value of $\alpha$ for the case of $\eta=0.20$ in Fig. 5, without any diurnal variation. As seen in Fig. 6, the STBL diurnal cycle in this case has the same incorrect phase as the one produced with $\eta=0.77$ (see Fig. 2), although the mean magnitudes of $h, b$ and $L$ are similar to those obtained with $\eta=0.20$ (see Fig. 3). Hence the entrainment parametrization LL98 with a low efficiency $\eta$ not only gives the right magnitude of the entrainment rate and its diurnal evolution, but also gives the right diurnal contribution 


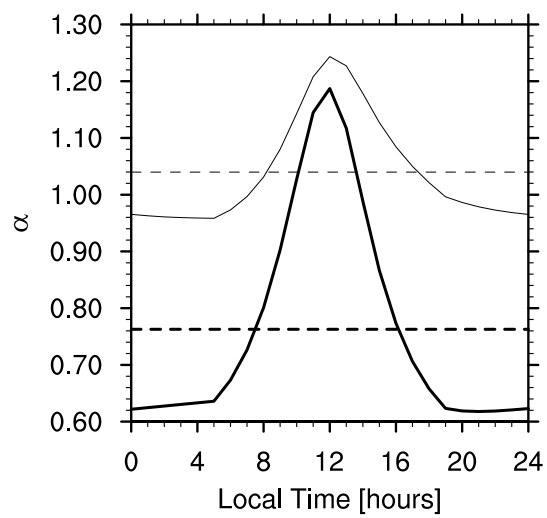

Figure 5. Diurnal variation in $\alpha$ (see text) and its mean value. Solid lines represent the diurnally varying $\alpha$, dashed lines its mean values; heavy lines for entrainment efficiency $\eta=0.20$; light lines for $\eta=0.77$.
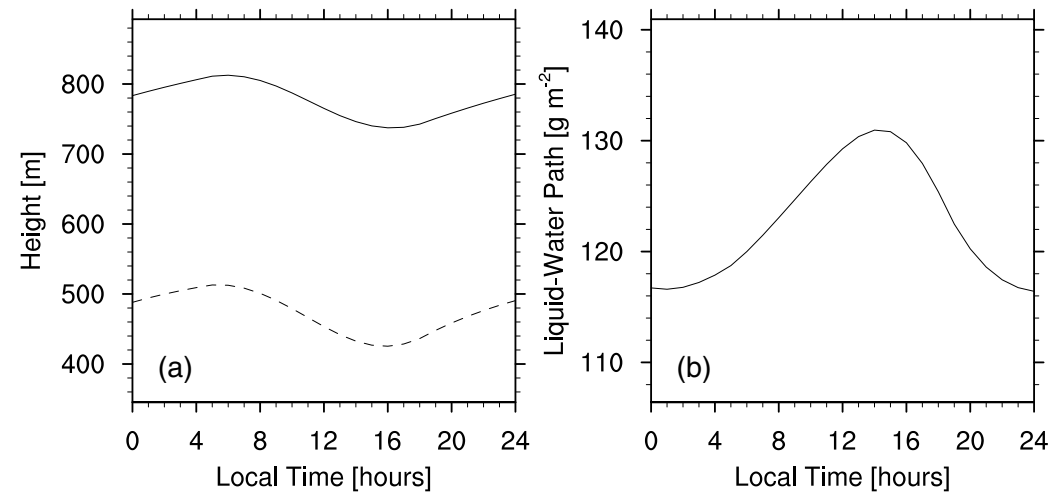

Figure 6. Diurnal variation of the STBL with constant $\alpha$ which equals the mean value in the case of $\eta=0.20$ (compare with Fig. 5). (a) Mixed-layer depth (solid), cloud-base height (dashed); (b) liquid-water path.

from the radiative driving to TKE generation. Indeed, the latter is the essential ingredient in the success of this parametrization.

The ratio of the radiative contribution to TKE production to the total TKE generation thus evolves diurnally. Besides radiation, surface forcing also contributes to the TKE budget and hence to cloud-top entrainment. Surface forcing becomes comparable to radiative driving when $\alpha>1$. At such high $\alpha$-values, decoupling between the cloud layer and the surface may indeed occur (Bretherton and Wyant 1997; Stevens 2000). In Fig. 5, $\alpha$ is greater than unity for only 4 hours when $\eta=0.20$, as compared to 12 hours when $\eta=0.77$. The cloud and surface layers are susceptible to decouple for a much shorter time with $\eta=0.20$ than with $\eta=0.77$. Such behaviour is consistent with the results of Turton and Nicholls (1987), i.e. their Fig. 11. To the extent that $\alpha>1$ is a measure of decoupling, these results help quantify the extent to which decoupling processes should be accounted for to explain the observed diurnal cycle.

In the present section, we have focused on how the diurnal STBL cycle depends on the entrainment efficiency embodied by the LL98 parametrization. By choosing the efficiency sufficiently large, we were able to capture the essence of the behaviour of the S76 entrainment rule. This particular rule was the source of misconceptions as to the utility of the MLM framework to study the diurnal cycle. A natural question is whether these 
results generalize to other entrainment parametrizations. They do. We have tested this assertion by repeating our analysis with the entire family of entrainment parametrizations evaluated by Stevens (2002); those parametrizations which entrain efficiently tend towards the behaviour evident in the S76 results (or LL98 with large $\eta$ ), while those that entrain less efficiently tend more towards the behaviour of LL98 with $\eta=0.20$. Small differences in detailed STBL behaviour arise from the way in which the efficiencies of different parametrizations evolve through the day, but these differences do not affect the main result of this section.

\section{SENSITIVITY OF CLOUD RADIATIVE PROPERTIES TO ENTRAINMENT EFFICIENCY}

In the previous section, we have examined in some detail the influence of entrainment efficiency on the diurnal variation of the liquid-water path $L$. This motivates us to ask if other important predictions of mixed-layer theory are also sensitive to entrainment efficiency.

In pristine maritime environments, small perturbations in aerosol concentrations can be reflected by changes in the number density $N$ of droplets. The influence of variations in $N$ on cloud albedo $A$ is measured by the susceptibility $S \equiv \mathrm{d} A / \mathrm{d} N$ (Twomey 1991; Platnick and Twomey 1994). Studies have shown $A$ to be a strong function of $L$ (e.g. Stephens 1978a). From the previous section, the equilibrium value of $L$, as well as its diurnal cycle, depend on $\eta$. Thus it is clear that, in the absence of other processes, the entrainment efficiency will affect the climatology of cloud radiative properties. Although different aspects of the susceptibility have been studied in the past (Pincus and Baker 1994; Platnick and Twomey 1994), it is interesting to revisit this question in light of the previous analysis. In other words, if one studies how cloud radiative properties depend on the aerosol concentration, how would one's results be affected by the model's entrainment parametrization?

A major component of $S$ is the well-known Twomey (1977) effect: given a constant $L, A$ increases with $N$ because smaller drops tend to have a larger total reflectivity. The Twomey effect is also sometimes called the first indirect effect of aerosols on atmospheric radiation. This, however, is not the whole story. Boers and Mitchell (1994) proposed an absorption feedback whereby variations in $N$ may lead to changes in solar absorption and hence on STBL dynamics, which cause further feedbacks on the radiative properties. By modulating drizzle, variations in $N$ may also affect the hydrological cycle of low clouds, in terms of both the horizontal extent (Albrecht 1989) and the vertical depth (Pincus and Baker 1994).

Besides the Twomey effect, varying $N$ thus influences $A$ indirectly through $L$. These latter effects are often called cloud lifetime effects or the second indirect effect. The first and second indirect effects can be summarized as follows:

$$
S=\left.\frac{\partial A}{\partial N}\right|_{L}+\left.\frac{\partial A}{\partial L} \frac{\partial L}{\partial N}\right|_{\eta}=S_{1}+S_{2},
$$

where subscript 1 and 2 stand for the first and second indirect effect, respectively. In what follows, $S$ is measured in the unit of 'percent $\mathrm{cm}^{3}$. . For example, $S=1$ means $A$ will increase from $50 \%$ to $51 \%$ for an increase in $N$ by $1 \mathrm{~cm}^{-3}$. Here we focus exclusively on the effect of aerosols in so far as they act as cloud condensation nuclei. Based on this, we calculate the reflectance and absorption of the cloud. This does not include the direct effect of aerosols or their optical properties.

To study the different components of $S$ requires the representation of precipitation and a more sophisticated treatment of radiative processes within the MLM of section 2. 
To parametrize the effect of drizzle on total-water specific humidity $q_{\mathrm{t}}$, we use the relationship proposed by vanZanten et al. (2005) from an analysis of DYCOMS-II data:

$$
\Delta F_{q}=3.4 \frac{(h-b)^{3}}{N} \text {. }
$$

In the implementation of (18), we neglect the sub-cloud evaporation of drizzle for simplicity. This underestimates the true effect of precipitation.

To better represent radiative processes, we couple the MLM to the radiation code of $\mathrm{Fu}$ and Liou (1992), which allows us to diagnose radiative fluxes at different vertical levels according to the vertical profiles of atmospheric characteristics such as temperature, humidity, ozone concentration and so on. The profiles inside the STBL are constructed from our MLM simulation results. Conditions between STBL top and $10 \mathrm{~km}$ are based on DYCOMS-II data. Above $10 \mathrm{~km}$ climatological values are used. By coupling the Fu-Liou code with the MLM, we are able to model the interactions between the diurnally varying cloud-top radiative driving and the STBL dynamics.

The environmental conditions are the same as in Table 1, except that $5 \times 10^{-6} \mathrm{~s}^{-1}$ is used for the large-scale divergence to insure the existence of cloudy solutions in the presence of the drizzle effect (18). Each simulation starts from an equilibrium where the STBL state and the radiative driving at cloud top are matched, but individual solutions vary with $N$ and $\eta$. Each simulation stops when the diurnal cycle stabilizes to an exact repetition. In what follows, we only discuss the results that are obtained by averaging such stabilized diurnal cycles over their daytime values.

For any given pair of $N$ and $\eta$ values, the total $S$ is obtained from two simulations as

$$
S(N, \eta)=\frac{A(N+\Delta N, \eta)-A(N-\Delta N, \eta)}{2 \Delta N},
$$

where $\Delta N=0.1 N$ and $A$ is a daytime average, as defined previously. The Twomey effect, $S_{1}(N, \eta)$, is obtained by diagnostically calculating the change in $A$ between $N+\Delta N$ and $N-\Delta N$ from the Fu-Liou code given the equilibrium state of STBL; $S_{2}$ is then simply $S-S_{1}$.

As previously mentioned, there are two components of $S_{2}$ : the absorption feedback effect proposed by Boers and Mitchell (1994) and the precipitation effect proposed by Pincus and Baker (1994). An additional set of simulations has been performed, therefore, with the drizzle parametrization disabled: $S_{2}$ is obtained as before, but because no drizzle is present it contains only the absorption feedback component. The first finding from these experiments is that the absorption feedback component is negligible and never exceeds $10 \%$ of the total $S_{2}$. Because the precipitation effect component dominates in $S_{2}$, in the following discussion we speak interchangeably of $S_{2}$ and the precipitation effect of Pincus and Baker (1994).

The results from interactive-radiation simulations are shown in Fig. 7. In Fig. 7(a), $L$ increases with $N$ because from (18) more droplets lead to less precipitation, while it decreases with $\eta$ because stronger entrainment leads to more vigorous warming and drying of the STBL. Similar behaviour is seen in Fig. 7(b) for cloud albedo because, for fixed $N, A$ is a non-decreasing function of $L$.

For small values of $N$, the drizzle flux is large so that most of the liquid water in the cloud is depleted and the variation in $L$ caused by more vigorous entrainment is damped. Thus, for small $N$, the water and heat budgets are both dominated by drying and warming due to the drizzle effect. For large $N$, however, the drizzle flux is small and so the entrainment warming and drying plays a dramatic role in the heat and water budgets. 
(a)

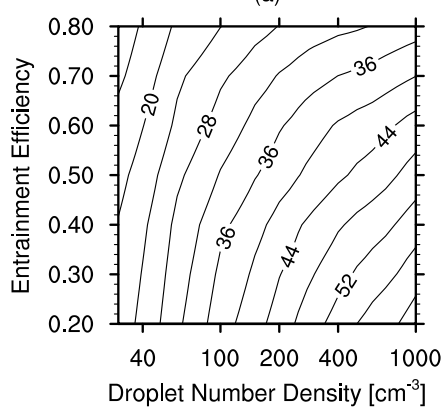

(b)

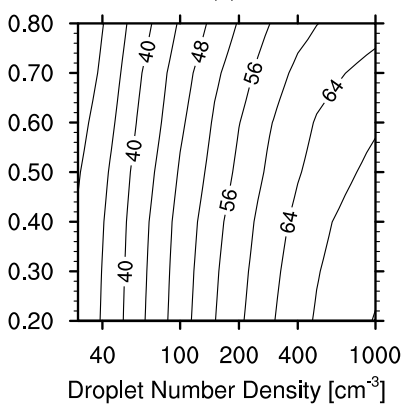

(c)

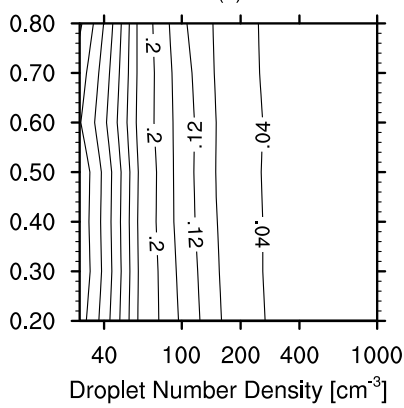

Figure 7. Contour plots of (a) liquid-water path $\left(\mathrm{g} \mathrm{m}^{-2}\right)$, (b) cloud albedo (percent), and (c) the total susceptibility (percent $\mathrm{cm}^{3}$ ), for different $N$ (along the $x$-axis) and $\eta$ (along the $y$-axis). All the results are from interactive radiation-MLM simulations. The $x$-axis uses a logarithmic scale.

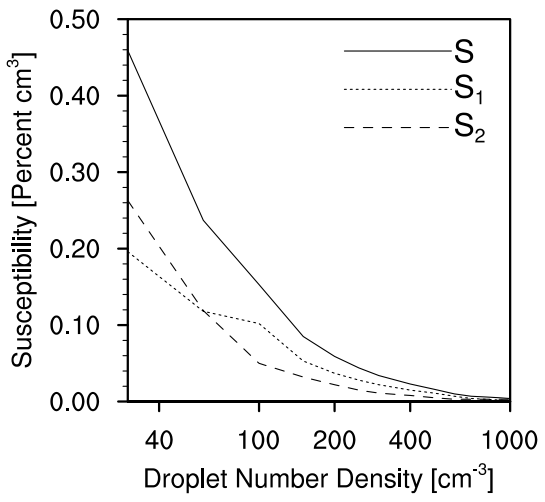

Figure 8. Dependence of the total susceptibility $S$, as well as the first and the second indirect effects, $S_{1}$ and $S_{2}$, on $N$, for entrainment efficiency $\eta=0.20$. Same scale on the abscissa as in Fig. 7.

Although both $L$ and $A$ show variation with $N$ as well as $\eta$, the total $S$ in Fig. 7(c) varies primarily with $N$ : there is little or no sensitivity of $S$ to $\eta$. The main variation in $S$ occurs for smaller $N$ values.

Figure 8 shows that the susceptibilities all decrease with $N$, with the greatest variation for $N<200 \mathrm{~cm}^{-3}$. Contributions from the first and second indirect effects are comparable. Although Fig. 8 is produced for $\eta=0.20$, Fig. 7 indicates that similar trends prevail for other values of $\eta$.

Why is $S$ more or less independent of $\eta$ ? At the smaller values of $N$, the drizzle warming and drying effect dominates; hence $L$ and $A$ themselves show much more variation with $N$ than with $\eta$. Thus we may expect that, for small $N, S$ is also fairly independent of $\eta$. At larger values of $N, L$ and $A$ show considerable variation with both $N$ and $\eta$. As $N$ becomes larger, however, $S$ itself asymptotes to 0 and hence can no longer vary much with $\eta$ either.

These arguments can be made more quantitative by noting that, to a good degree of approximation,

$$
\begin{aligned}
\tau & =0.19 L^{5 / 6} N^{1 / 3}, \\
A & =\frac{\tau}{6.8+\tau},
\end{aligned}
$$


(a)

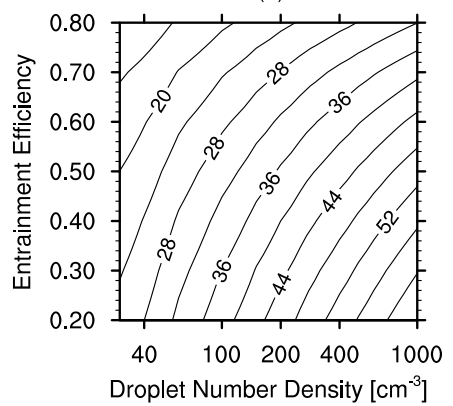

(b)

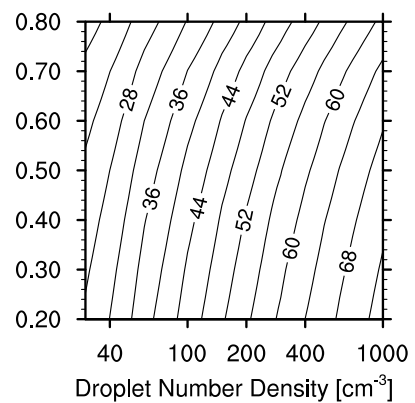

(c)

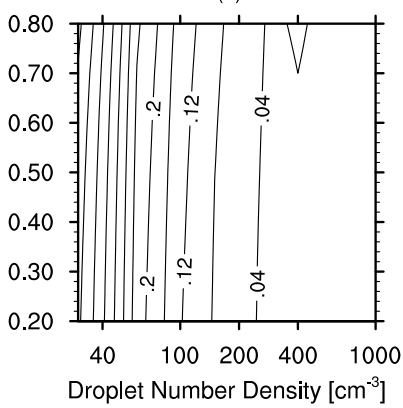

Figure 9. Same as Fig. 7, except using the analytical semi-empirical formulae (20)-(23).

where $\tau$ displayed as in (19) is the cloud optical depth. Equation (19) follows Stephens (1978b) for the case where $N$ is constant and $q_{\ell}$ increases linearly with the height above cloud base. Our expression (20) for $A$ follows Lacis and Hansen (1974). The constant 6.8 is chosen to fit our data and can also be related to the asymmetry factor for the cloud particle phase function. To get the corresponding expression for $S$, we need to specify the dependence of $L$ on $N$ and $\eta$. The following relationship provides a good fit to the data in Fig. 7(a):

$$
L=12(1-\eta)^{0.5} \ln (N / 3) .
$$

Equation (21) implies that the effect of $\eta$ on $L$ increases as $N$ becomes larger.

With the help of these expressions, it is straightforward to show that:

$$
S_{1}=2.3 \frac{A^{2}}{\tau N}, \quad S_{2}=5.7 \frac{A^{2}}{\tau N}\left(\ln \frac{N}{3}\right)^{-1},
$$

and hence that

$$
S=\frac{0.19 L^{5 / 6} N^{1 / 3}}{\left(6.8+0.19 L^{5 / 6} N^{1 / 3}\right)^{2}}\left(\frac{2.3}{N}+\frac{5.7}{N \ln N / 3}\right) .
$$

On the right-hand side of (23), the second factor is a strictly decreasing function of $N$. The dependence of $S$ on $\eta$ resides in $L$ in the first factor, according to (21); the factor as a whole also decreases with $N$. When $N$ is small, $S$ is relatively large (see Fig. 8), but the variation in $L$ caused by $\eta$ is not pronounced. When $N$ is large, the variation in $L$ caused by $\eta$ becomes apparent, but $S$ itself is so small that it never exceeds $10 \%$ of its value at small $N$. This behaviour is evident in Fig. 9, in which $L, A$ and $S$ are analytically calculated, based on the semi-empirical formulae introduced earlier. Comparing Fig. 9 with Fig. 7, we find that the results are quite similar.

The analysis above, together with Fig. 9, helps justify our explanation of Fig. 7, namely that the susceptibility is relatively insensitive to the entrainment efficiency as long as the simulations permit cloudy solutions. The implication is that, while the choice of the entrainment closure for the MLM may lead to different estimates of liquid-water path and cloud albedo, the susceptibility of cloud albedo to aerosol concentration will not be greatly affected.

Although our purpose has been to investigate how estimates of susceptibility depend on one's entrainment closure, it is also interesting to compare the numerical values of susceptibility to previously published values. Such a comparison is facilitated 
by writing (23) as $S=S_{1}(1+\delta)$, where $\delta$ is equivalent to the radiative enhancement factor in Eq. (5) of Pincus and Baker (1994), or $2 \beta$ in Eq. (9) of Platnick and Twomey (1994). For the case $N=100 \mathrm{~cm}^{-3}, \delta=0.71$ in our study, as compared to $2 \beta=0.84$ for Platnick and Twomey (1994) and $\delta=1.5$ for Pincus and Baker (1994). These values are of comparable magnitude, which suggests that insights from those earlier studies are broadly applicable to studies based on modestly different representations of microphysical processes, as discerned from the DYCOMS-II data.

\section{CONCLUSIONS}

We examined the diurnal cycle of the stratocumulus-topped boundary layer and the susceptibility of its cloud albedo to changes in droplet number density. Our study emphasized the sensitivity of the mixed-layer representation of the STBL to the model's entrainment efficiency $\eta$. We find that the diurnal cycle is accurately modelled for weak entrainment efficiencies and that the susceptibility is rather insensitive to the entrainment efficiency.

The marked sensitivity of the diurnal cycle to $\eta$ is found to depend on the equilibrium heights of cloud boundaries for a prescribed diurnally varying radiative driving, as well as on the time-scales at which these heights adjust to their equilibrium values. The adjustment time-scale for cloud-top height is determined primarily by large-scale processes, i.e. the large-scale subsidence; the time-scale for cloud base is determined mainly by the turbulent processes responsible for the fluxes of heat and water. In typical circumstances, the former is much longer than the latter, i.e. roughly one week vs. one day (Schubert et al. 1979).

In the case of high entrainment efficiencies, a pronounced diurnal variation is found in the instantaneous equilibria of both cloud boundaries. This behaviour results in comparable differences, for both boundaries, between the results for cloud boundary height from a time-dependent MLM calculation and its equilibrium value. Because of the distinct adjustment time-scales, however, the descent rate of the cloud top is less than the one of the cloud base, which leads to cloud thickness growing unrealistically during the daytime.

In the case of low entrainment efficiencies, a pronounced diurnal variation is found in the equilibrium height of cloud top but not in cloud base. Hence the descending behaviour of the cloud top governs the daytime evolution of cloud thickness, irrespective of the adjustment time-scales, which results in a much more realistic STBL diurnal cycle.

Having defined a radiative entrainment efficiency $\alpha$, we further explored how different entrainment efficiencies may influence the equilibria of the STBL. By examining $\alpha$, we found that only low entrainment efficiencies produce a diurnal variation in the contribution of the radiative driving to TKE generation; this turns out to be a key element in producing the right diurnal cycle of the cloud thickness.

In the susceptibility study, both the first and second indirect effects of aerosols on cloud albedo are pronounced and comparable in magnitude. The first of these (Twomey 1977) is associated with variation in cloud albedo due to the change of droplet number density $N$ given constant liquid-water path $L$. The second one (Albrecht 1989; Boers and Mitchell 1994; Pincus and Baker 1994) is mediated by the effects of $N$ on liquidwater path $L$. The precipitation process associated with drizzle (Pincus and Baker 1994) dominates in the second indirect effect.

Different entrainment efficiencies $\eta$ lead to different estimates of liquid-water path and cloud albedo; still, the susceptibility of cloud albedo to the changes in droplet 
number density $N$ is only weakly affected by these estimates. This result is important and has therefore been verified both by numerical simulation, using a fully interactive MLM-radiation model, and by an analytical derivation that relied on approximate, semiempirical fits to the simulation results. Our conclusions in this respect are encouraging for climate-sensitivity studies aimed at quantifying the susceptibility of stratocumulus albedo to perturbations in $N$.

In summary, a better diurnal variation of cloud thickness can be produced by mixed-layer theory when using low entrainment efficiencies. The success of an MLM under these circumstances suggests that diurnal decoupling between the cloud layer and the surface may be less important than previously thought. The independence of total susceptibility $S$ with respect to entrainment efficiency $\eta$ is beneficial for climatesensitivity studies with perturbed aerosol concentrations, especially when models with uncertain entrainment physics are used to parametrize the STBL. These results should be of great interest to the climate modelling community, especially those that have MLM as their boundary-layer parametrization in the stratocumulus regime.

\section{ACKNOWLEDGEMENTS}

We would like to acknowledge Kuo Nan Liou and $\mathrm{Yu} \mathrm{Gu}$ for the radiation code. It is a pleasure to thank three anonymous reviewers for numerous and constructive comments. Michael Ghil and Yunyan Zhang were partially supported by the National Science Foundation, Grant \# ATM00-82131, while Bjorn Stevens was supported by the National Science Foundation, Grant \# ATM-9985413.

Albrecht, B. A.

Boers, R. and Mitchell, R. M.

Bretherton, C. S. and Wyant, M. C.

Bretherton, C. S., Uttal, T., Fairall, C. W., Yuter, S. E., Weller, R. A.,

Baumgardner, D., Comstock, K., Wood, R. and Raga, G. B.

Duynkerke, P. G., de Roode, S. R., vanZanten, M. C., Calvo, J., Cuxart, J., Cherinet, S., Chlond, A., Grenier, H., Jonker, P. J., Köhler, M., Lenderink, G., Lewellen, D., Lappen, C., Lock, A., Moeng, C.-H., Müller, F., Olmeda, D., Piriou, J.-M., Sanchez, E. and Sednev, I. $\mathrm{Fu}, \mathrm{Q}$. and Liou, K. N.

Grenier, H. and Bretherton, C. S.

Hignett, P.

Lacis, A. A. and Hansen, J. E.

\section{REFERENCES}

1989

1994

1997

2004

2004

\begin{abstract}
Observations and numerical simulations of the diurnal cycle of the EUROCS stratocumulus case. Q. J. R. Meteorol. Soc., 130 , 3269-3296
\end{abstract}

Aerosols, cloud microphysics and fractional cloudiness. Science, 245, 1227-1230

Absorption feedback in stratocumulus clouds influence on cloud top albedo. Tellus, 46A, 229-241

Moisture transport, lower-tropospheric stability, and decoupling of cloud-topped boundary layers. J. Atmos. Sci., 54, 148-167

The EPIC 2001 stratocumulus study. Bull. Am. Meteorol. Soc., 85, 967-977
On the correlated K-distribution method for radiative-transfer in nonhomogeneous atmospheres. J. Atmos. Sci., 49, 2139-2156

A moist PBL parameterization for large-scale models and its application to subtropical cloud-topped marine boundary layers. Mon. Weather Rev., 129, 357-377

Observations of diurnal variation in a cloud-capped marine boundary layer. J. Atmos. Sci., 48, 1474-1482 earth's atmosphere. J. Atmos. Sci., 31, 118-133 
Lewellen, D. and Lewellen, W.

Lilly, D. K.

Liou, K. N.

Lock, A. P.

Pincus, R. and Baker, M. B.

Platnick, S. and Twomey, S.

Schubert, W. H.

Schubert, W. H., Wakefield, J. S., Steiner, E. J. and Cox, S .K. Stephens, G. L.

Stevens, B.

Stevens, B., Lenschow, D. H., Faloona, I., Moeng, C-H., Lilly, D. K., Blomquist, B., Vali, G., Bandy, A., Campos, T., Gerber, H., Haimov, S., Morley, B. and Thorton, D.

Stevens, B., Lenschow, D. H., Vali, G., Gerber, H.,

Bandy, A., Blomquist, B., Brenguier, J.-L.,

Bretherton, C. S., Bernet, F., Campos, T., Chai, S.,

Faloona, I., Friesen, D., Haimov, S., Laursen, K., Lilly, D. K., Loehrer, S. M., Malinowski, S. P., Morley, B., Petters, M. D., Rogers, D. C., Russell, L., Savic-Jovcic, V., Snider, J. R., Straub, D.,

Szumowski, M. J., Takagi, H., Thornton, D. C., Tschudi, M., Twohy, C., Wetzel, M. and vanZanten, M. C.

Turton, J. D. and Nicholls, S.

Twomey, S.

vanZanten, M. C., Duynkerke, P. G. and Cuijpers, J. W. M.

vanZanten, M. C., Stevens, B., Vali, G. and Lenschow, D. H. Vernon, E. M.
1998 Large-eddy boundary layer entrainment. J. Atmos. Sci., 55, 2645-2665

1968 Models of cloud topped mixed layers under a strong inversion. Q. J. R. Meteorol. Soc., 94, 292-309

2002 An introduction to atmospheric radiation. 2nd edition. Academic Press, San Diego, USA

2001 The numerical representation of entrainment in parameterizations of boundary layer turbulent mixing. Mon. Weather Rev., 129, $1148-1163$

1994 Effect of precipitation on the albedo susceptibility of clouds in the marine boundary layer. Nature, 372, 250-252

1994 Determining the susceptibility of cloud albedo to changes in droplet concentration with the advanced very high resolution radiometer. J. Appl. Meteorol., 33, 334-347

1976 Experiments with Lilly's cloud-topped mixed layer model. J. Atmos. Sci., 33, 436-446

1979 Marine stratocumulus convection. Part II: Horizontally inhomogeneous solutions. J. Atmos. Sci., 36, 1308-1324

1978 a Radiation profiles in extended water clouds. I: Theory. J. Atmos. Sci., 35, 2111-2122

1978b Radiation profiles in extended water clouds. II: Parameterization schemes. J. Atmos. Sci., 35, 2123-2132

2000 Cloud transitions and decoupling in shear-free stratocumulustopped boundary layers. Geophys. Res. Lett., 27, 2557-2560

2002 Entrainment in stratocumulus topped mixed layers. Q. J. $R$. Meteorol. Soc., 128, 2663-2690

2003a On entrainment rates in nocturnal marine stratocumulus. Q. J. R. Meteorol. Soc., 129, 3469-3493

2003b Dynamics and Chemistry of Marine StratocumulusDYCOMS-II. Bull. Am. Meteorol. Soc., 84, 579-593

A study of the diurnal variation of stratocumulus using a multiple mixed layer model. Q. J. R. Meteorol. Soc., 113, 969-1009

The influence of pollution on the shortwave albedo of clouds. J. Atmos. Sci., 34, 1149-1152

Aerosols, clouds and radiation. Atmos. Environ., 25A, 2435-2442

Entrainment parameterization in convective boundary layers. J. Atmos. Sci., 56, 813-828

2005 Observations of drizzle in nocturnal marine stratocumulus. J. Atmos. Sci., 62, 88-106

1936 The diurnal variation in ceiling height beneath stratus clouds. Mon. Weather Rev., 64, 14-16 